\title{
Introduction
}

\section{Biomarkers in multiple sclerosis}

\author{
Roland Martin ${ }^{\mathrm{a}}$, Bibiana Bielekova ${ }^{\mathrm{b}}$, Reinhard Hohlfeld ${ }^{\mathrm{c}}$ and Ursula Utz ${ }^{\mathrm{d}, *}$ \\ ${ }^{a}$ Catalan Institute for Research and Advanced Studies and Unidad de Neuroimmunologia Clinica, Hospital \\ Universitari Vall d'Hebron, Barcelona, Spain \\ ${ }^{\mathrm{b}}$ Department of Neurology, Waddel Center for MS, University of Cincinnati \& The Neuroscience Institute, \\ Cincinnati, $\mathrm{OH}, \mathrm{USA}$ \\ ${ }^{\mathrm{c}}$ Department of Neuroimmunology, Max-Planck-Institute of Neurobiology, Martinsried, Munich, Germany \\ ${ }^{\mathrm{d}}$ Division of Extramural Research, National Institute of Neurological Disorders and Stroke, National Institutes of \\ Health, Bethesda, MD, USA
}

Several processes are presumed to sequentially or simultaneously contribute to the pathophysiology of multiple sclerosis (MS). Biomarkers indicative of these processes would hold great potential for (1) MS diagnostics and identification of disease stages and subcategories; (2) prediction of onset and disease course; (3) treatment selection and improved prognosis of treatment success; and (4) the evaluation of novel therapeutics. Though it is unlikely that any one marker could function as a true surrogate or stand alone, biomarker combinations or patterns could provide insight into the mechanism of action of a drug and could suffice for the pre-screening of prospective therapeutics. To examine the potential of biomarkers in the context of MS, the National Institute of Neurological Disorders and Stroke (NINDS), NIH, organized a workshop "Biomarkers in Multiple Sclerosis”, held from April 14-16, 2004. Organized by the authors, an international group of participants including basic and clinical researchers, industry and FDA representatives and NIH staff explored the current state of biomarker research for MS, barriers to progress, possible solutions and priorities. The group explored biomarkers relevant to disease processes such as inflammation, axonal damage, demyelination, oxidative stress, and remyelination and evaluated individual biomarkers and biomarker approaches on their

${ }^{*}$ Corresponding author: Ursula Utz, Ph.D., National Institute of Neurological Disorders and Stroke, NIH, Neuroscience Center, 6001 Executive Blvd., Bethesda, MD 20892, USA. Tel.: +1 301496 1431; Fax: +1 301480 2424; E-mail: utzu@ninds.nih.gov. usefulness for the advancement of categories 1 to 4 listed above. This special edition of the Journal Disease Markers contains articles selected from presentations given at the meeting and provides a general overview and conclusions of the workshop.

Multiple sclerosis (MS) is a life-long disease, which initially takes a fluctuating course with unpredictable exacerbations, usually followed by complete or partial recovery (relapsing-remitting phase). After several years, the course becomes for most patients relentlessly progressive (secondary progressive phase). In a minority of patients, the disease presents with progressing neurological deficits without relapses from the beginning (primary progressive form of MS). The many features of MS complicate an assessment of actual activity of disease. Clinical symptoms of MS can be highly subjective, making it difficult to objectify, let alone quantify them. To exemplify, serial magnetic resonance imaging (MRI) studies demonstrated that active inflammatory lesions don't necessarily translate into clinical signs or symptoms.

A number of immunomodulatory and - suppressive treatments, including three preparations of interferon$\beta$, glatiramer acetate, and mitoxantrone, are currently available for MS therapy. These therapies work only long-term, and seem to require life-long application. Although these medications dampen disease activity and ameliorate its clinical course to a certain extent, they do not cure disease. The unpredictable and chronic nature of MS and the difficulty to objectify its clinical course, pose substantial problems in assessing whether 
any immunomodulatory treatment is effective in individual patients. Usually a year of continuous treatment is required before the treatment effect can be judged.

Matters are further complicated by an increasing evidence for MS being a heterogeneous disease. At least four histopathological subtypes of acute demyelination have been described [5], and additional subtypes, such as optico-spinal MS (Neuromyelitis optica (NMO)), were distinguished using clinical and/or laboratory features. Besides the pathophysiological mechanisms that cause acute demyelination, susceptibility to central nervous system (CNS) damage and repair impairment are factors that contribute to the severity of immune-mediated insult and are likely to influence the development of disability.

For these reasons, para-clinical markers are needed to assess disease activity, distinguish disease subtypes, provide prognostic information, and distinguish clinical responders from non-responders to immunomodulatory treatment. The term "biomarker" has been defined as "a characteristic that is objectively measured and evaluated as an indicator of normal biologic processes, pathogenic processes, or pharmacologic responses to a therapeutic intervention" $[3,4]$. In contrast, "surrogate endpoint" defines a biomarker that is intended to serve as a substitute for a clinically meaningful endpoint and is expected to predict the effect of a therapeutic intervention or the evolution of disease. The use of the term "surrogate marker" is discouraged [1]. "Clinical endpoint" defines a meaningful measure which captures how a patient feels, functions or survives. Clinical endpoints may be further classified as a) "intermediate endpoint", which represents a clinical endpoint that is not the ultimate outcome but is nonetheless of real clinical usefulness (e.g. exacerbation rate in relapsing-remitting MS); and b) "ultimate clinical outcome", which is a clinical endpoint reflective of accumulation of irreversible morbidity and survival (e.g. accumulation of disability in MS) [2]). For MS, the most useful biomarkers to date are those related to magnetic resonance imaging (MRI) of the CNS which allows the identification and quantification of MS lesions in an objective and increasingly sophisticated manner. Tremendous progress has been made in the field of MS imaging, and MRI measurements have long become an essential component of MS diagnostic and therapeutic trials. Nevertheless, MR imaging still has many limitations, including its relatively low sensitivity and a lack in understanding which imaging measures reflect what types of pathogenetic events such as immunopathological processes. Together with the complexity of the disease this explains the rather poor correlations between currently available MRI measures and the progression of clinical disability in MS.

This unsatisfactory situation led to an intense and ongoing search for new biologic markers that are independent and distinct from imaging markers. These efforts are greatly aided by the enormous power of novel technologies developed as "toolboxes" for the study of molecular immunology and genetics, including the famous "omics" (genomics, proteomics, metabolomics). The availability of these effective instruments creates novel opportunities for the emerging field of MS biomarkers. The NINDS workshop on "Biomarkers in multiple sclerosis" provided a forum for basic and clinical researchers in MS and related fields, and for industry and FDA representatives to explore the current state of biomarker research in MS, the barriers to progress, and possible solutions and priorities. Although biomarkers can encompass parameters other than biological molecules such as susceptibility genes, imaging measures and e.g. neurocognitive test results, the organizers saw a particular need to focus on gene expression and disease-correlated fluctuations of biological molecules including but not limited to proteins, peptides, antibodies, radicals, and lipids. Biomarkers relevant to inflammation, axonal damage, demyelination, oxidative stress, and remyelination were discussed as were technologies for their identification and quantification and the applicability of biomarker discovery to a variety of clinical aims. The workshop began with an introduction into MS disease pathogenesis by Reinhard Hohlfeld and a statement of the workshop goals by Roland Martin. These presentations were followed by a discussion of biomarker versus surrogate endpoint by Art Atkinson which defined the latter as a substitute for a clinically meaningful endpoint that would accurately predict the effect of a therapeutic intervention. Talks on the role of biomarkers in therapeutic development programs in general (Marc Walton) and for MS specifically (Bibiana Bielekova) introduced presentations on MS biomarker categories. The discussed examples included neuroimaging (Douglas Arnold), biomarkers indicative of peripheral activation (Richard Ransohoff, Thomas Berger and Samia Khoury), of blood brain barrier disruption (Emmanuelle Waubant), and of lesion formation and repair (Gavin Giovannoni and Peter Werner). Day 2 concluded with presentations on biomarker discovery for cancer ((Sudhir Srivastava) and amyotrophic lateral sclerosis (Robert Bowser) and technologies for biomarker discovery such as antigen and DNA microarrays, proteomics and SNP analysis 
(Larry Steinman, Dietrich Stephan, Aaron Kantor, and David Hafler). The last day was dedicated to discussion in three break-out groups: 1) Diagnosis (MS categorization and disease course prediction); 2) Treatment (Drug screening and treatment outcome prediction); and 3) Making it Happen (sample types, collection methods and storage, data collection, assay standardization and validation).

The meeting concluded with the following observations and recommendations.

The meeting participants agreed that validated biomarkers would be invaluable for many aspects of MS and could aid in (1) MS diagnostics and identification of disease stages and subcategories; (2) prediction of onset and disease course; (3) treatment selection and improved prognosis of treatment success; and (4) the evaluation of novel therapeutics. Though it will be unlikely that any one marker could function as a stand alone, biomarker combinations or patterns could serve in achieving some of these aims. In an age of approved therapies for MS, placebo-controlled trials become ethically questionable. Trials of novel therapeutics conducted as add-on studies are expected to produce smaller treatment effects and will require larger samples sizes and/or a longer trial duration, both leading to cost increases for already costly trials. The panel felt that biomarkers will gain increasing importance in the pre-screening of prospective therapeutics, but acknowledged that currently there is no biomarker that could serve this purpose with the potential exception of neuroimaging. Biomarkers that predict therapy responses or identify disease subtypes might be most in reach and would serve important purposes such as the reduction of treatment failures and increase of statistical power for clinical trials via educated patient stratification. Biomarkers that help with de novo MS diagnostic rather than the identification of MS subtypes or of misdiagnosed patients might be harder to come by as will biomarkers that would predict disease progression. True surrogate endpoints that could replace clinical outcome measures as primary endpoints for clinical trials will not be available for years to come if ever. Also, due to the complexity of the disease process and disease heterogeneity in MS, it is unlikely that a single biomarker will be able to serve as marker for any of the above clinical aims.

The group saw a need for both hypothesis- and discovery-driven approaches to biomarker discovery. Some of the presented technologies and their potentials raised enthusiasm about discovery-driven approaches which by some were seen as less hampered by pre- conceived notions about disease pathology. Unexpected "hits" could lead to innovative therapeutic approaches targeting novel disease pathways.

To achieve economies of scale, the participants suggested that biomarker data would best be gathered in conjunction with a clinical trial. Biomarker discovery requires comparable phenotypic characterizations, logistics and statistical analyses. It was even suggested that every treatment trial include a biomarker component and the panel warned that not collecting samples would signify missed opportunities. Sample repositories and data banks will be needed as will be a validation of assay systems. The NIAID Immune Tolerance Network and the NCI Early Detection Research Network were discussed in this context. Such sample and data repositories should reflect national and international collaborations and should provide free access. Intellectual property issues for biomarker data gathered in conjunction with industry-sponsored trials will need to be resolved. Finally, an appeal was made for funding agencies to provide mechanisms that would accommodate the requirements of biomarker proposals. A shortening of the review cycle to allow piggybacking with a parent clinical trial was discussed as were special study sections with expertise and appreciation for biomarker studies. Currently, proposals to collect samples and data during a natural history study or a treatment trial are often criticized and rejected as lacking a clear hypothesis.

The meeting closed on the optimistic notion that great progress is to be expected in the near term future and that the development and improvement of new technologies will greatly aid in the search for biomarkers for MS.

\section{References}

[1] NIH-FDA Conference: Biomarkers and Surrogate Endpoints: Advancing Clinical Research and Applications. Abstracts, Dis Markers 14(4) (1998), 187-334.

[2] B. Bielekova and R. Martin, Development of biomarkers in multiple sclerosis, Brain 127(Pt 7) (2004), 1463-1478.

[3] E. Floyd and T.M. McShane, Development and Use of Biomarkers in Oncology Drug Development, Toxicol Pathol 32(Supplement 1) (2004), 106-115.

[4] L.J. Lesko and A.J. Atkinson, Jr., Use of biomarkers and surrogate endpoints in drug development and regulatory decision making: criteria, validation, strategies, Annu Rev Pharmacol Toxicol 41 (2001), 347-366.

[5] C. Lucchinetti, W. Bruck et al., Heterogeneity of multiple sclerosis lesions: implications for the pathogenesis of demyelination, Ann Neurol 47(6) (2000), 707-717. 


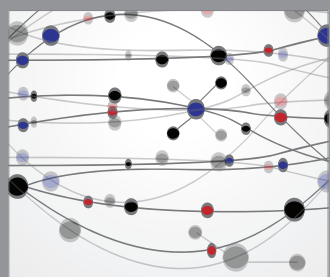

The Scientific World Journal
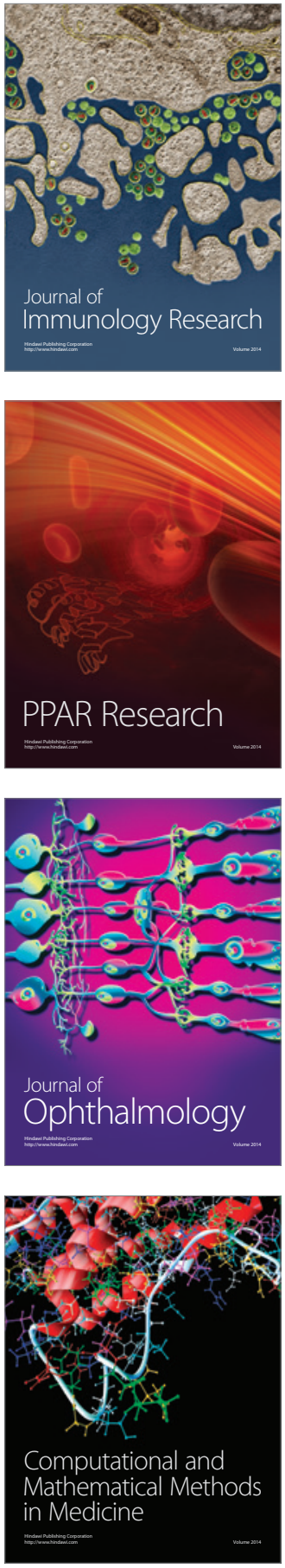

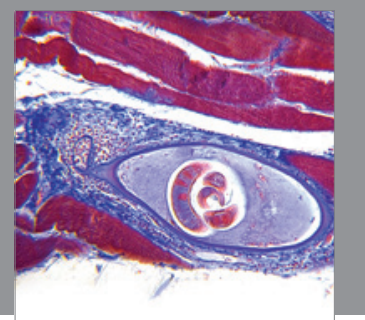

Gastroenterology

Research and Practice
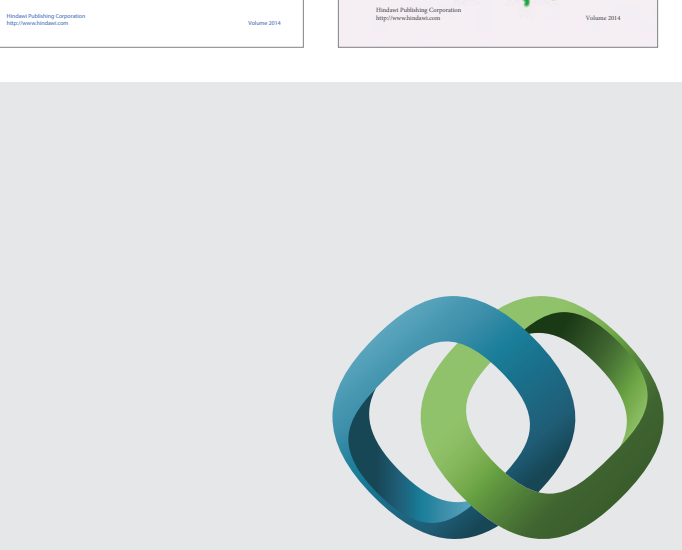

\section{Hindawi}

Submit your manuscripts at

http://www.hindawi.com
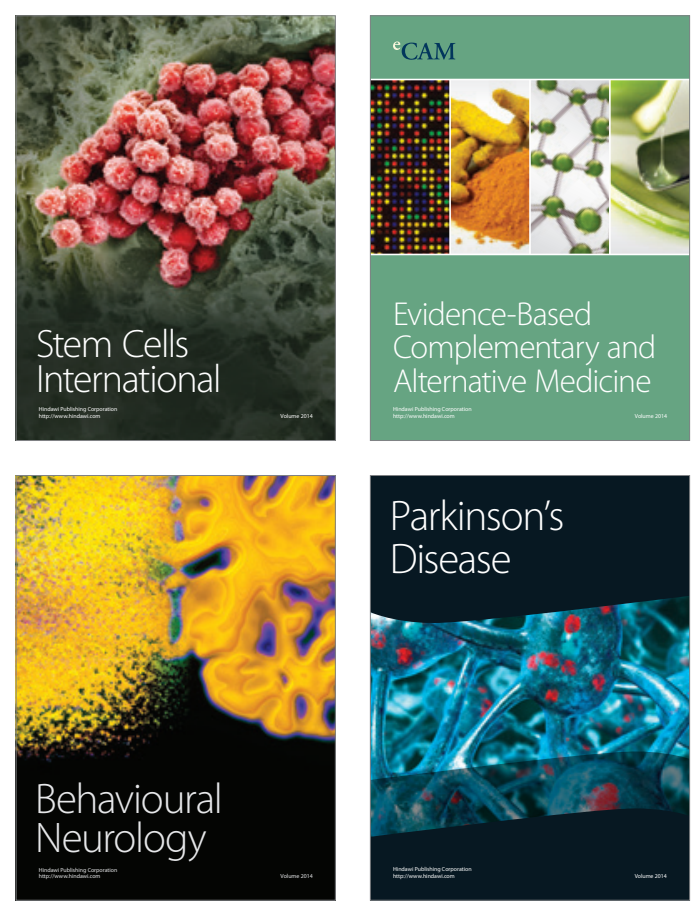

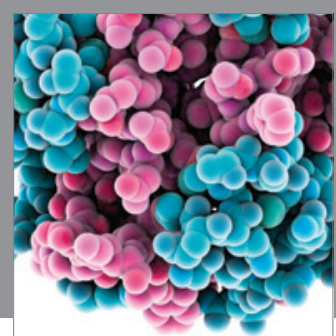

Journal of
Diabetes Research

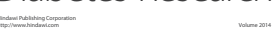

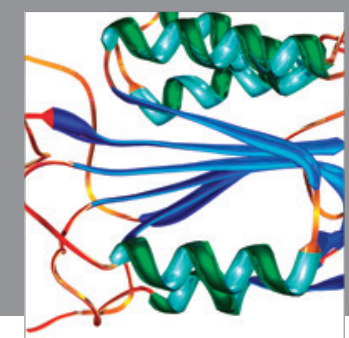

Disease Markers
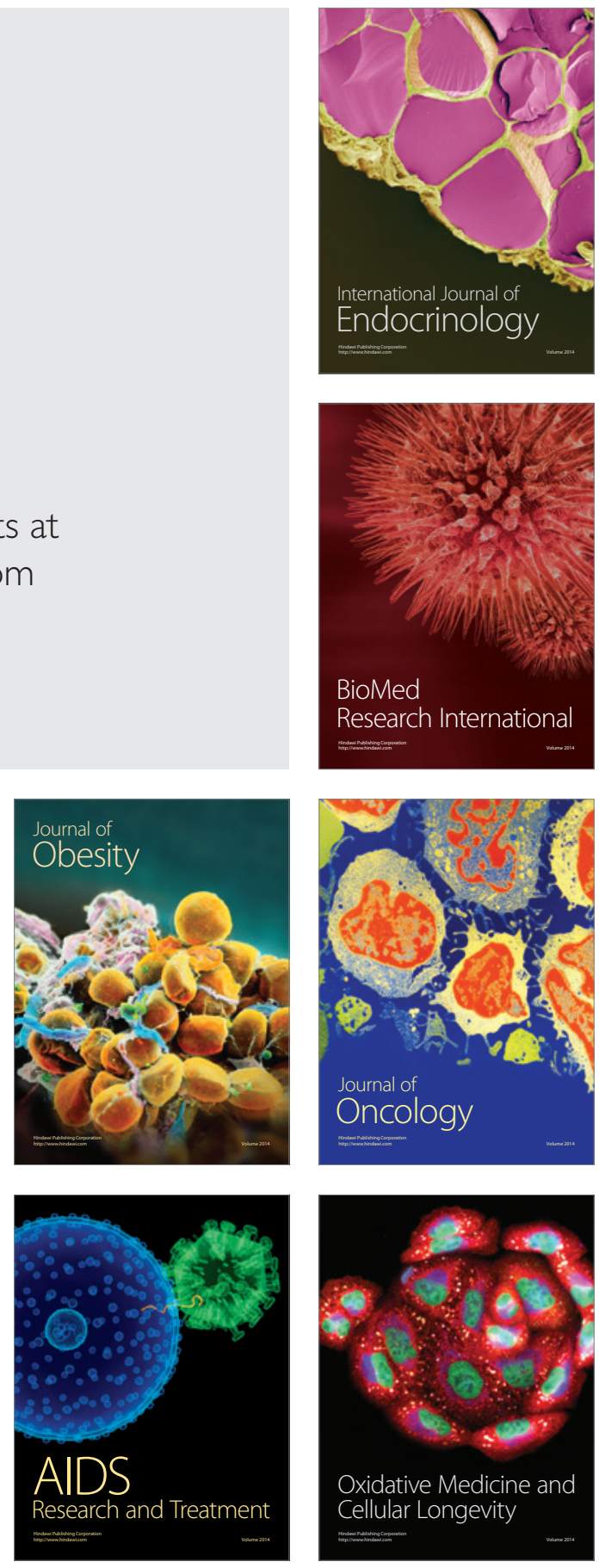